\title{
Redshift Determination for Quasar Based on Similarity Measure
}

\author{
Fuqing Duan and Fuchao Wu \\ National Laboratory of Pattern Recognition, \\ Institute of Automation, Chinese Academy of Sciences, \\ P.O.Box 2728,Beijing P.R.China,100080 \\ ffqduan, fcwu\}@nlpr.ia.ac.cn
}

\begin{abstract}
With the advent of very large redshift surveys, automatic redshift measurement is becoming increasingly important. This paper presents a similarity measure based cross-correlation method for the redshift determination of quasar spectra. Cross-correlation is measured only for the redshift candidates that are determined by the emission line features of the observed spectrum. The similarity measure is defined as the weighted sum of several similarity evidences. Compared with the traditional cross-correlation based methods, our method can be used for higher redshift determination. Compared with the methods based on spectral line matching, our method is less sensitive to the quality of spectral line extraction. Experiment results indicate the high performance of the method.
\end{abstract}

\section{Introduction}

The development of fiber-based spectrographs capable of observing hundreds of celestial objects simultaneously has led to many large redshift surveys such as SDSS, 2DF, LAMOST etc. Because of the sheer size of these surveys, it is becoming very important to develop methods of reliable and automated spectral recognition. An astronomical spectrum consists of continuum, spectral lines and noises. The continuum is produced by continuous radiation along wavelength, and it is the low frequency ingredients of a spectrum. Spectral lines include absorption lines and emission lines, which are produced by some atoms in celestial objects for absorbing or radiating energy at fixed wavelengths. An observed spectrum is shown in fig.1, where the horizontal axis denotes wavelength, the vertical axis represents relative flux, the smooth thick curve denotes the continuum, and the spectral lines are indicated. Because of the movement away from the earth of celestial objects, the wavelengths of spectral lines in observed spectra are usually larger than those in the rest frames. That is the redshift phenomenon. Redshift is one of the most important parameters of celestial objects. It can be computed by the formula $Z=\left(\lambda-\lambda_{0}\right) / \lambda_{0}$, where $\lambda$ stands for the wavelength of a spectral line in the observed spectrum, $\lambda_{0}$ for its corresponding wavelength in the rest frame, Z for redshift. Quasars (QSO) are the brightest and furthest celestial objects detected up to now. They play a very important role in the search of the 
universe, and are given more and more attention. Redshift determination of an observed QSO spectrum is a main task in QSO recognition.

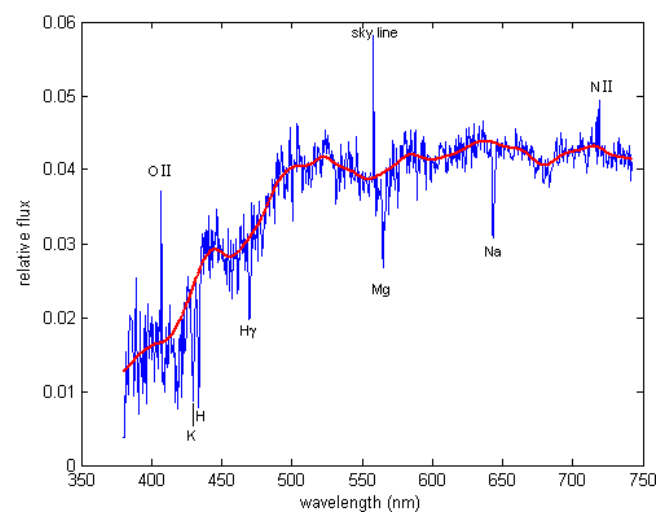

Fig. 1. An observed spectrum

The observed QSO spectra have the following characters: firstly, the spectra are usually contaminated heavily by noise so that the spectra vary drastically from their rest frames; secondly, the observed wavelength range is limited, generally from $370 \mathrm{~nm}$ to $780 \mathrm{~nm}$ about, which is a part of overall wavelength of QSO; finally, there are very few spectral lines in most spectra for their large redshifts. These characters make it very difficult to determine the redshift automatically.

Existing automated methods for redshift determination can be divided into two classes. The one is based on cross-correlation $[1,2]$, the other is based on spectral line matching. The most common technique [1] of the first class is the crosscorrelation of the observed spectrum with a set of templates, and the redshift is determined by the location of the largest peak in the cross-correlation functions. PCAZ [2] generalizes the cross-correlation approach by replacing the individual templates with a simultaneous linear combination of orthogonal templates gained by using principle component analysis (PCA). Although these methods are regarded as the most successful methods of automatic redshift measurement, unfortunately, they can be used only for those galaxy spectra with small redshifts. The typical methods in the second class include the approaches of Hough transform based [3] and density estimation based [4]. In these methods, spectral line extraction is a key step. However, due to aforementioned characters of the QSO spectra, the result of spectral line extraction usually is not satisfactory so that the result of redshift determination is not reliable. In order to reduce the dependence on the spectral line extraction in redshift determination, this paper presents a novel cross-correlation method by combining the two classes of methods. It determines the redshift candidates by the result of the spectral line extraction firstly, and then measures the cross-correlation for the redshift candidates. The novelty of the proposed method includes the following: 
1. Cross-correlation is measured only for the redshift candidates.

2. A new similarity measure is proposed, it can measure the similarity between two spectra more reasonably.

3. The new thresholding in feature extraction can improve the quality of spectral line extraction.

The remainder of this paper is organized as follows. Section 2 introduces the preprocessing of spectra. Section 3 introduces the feature extraction. Section 4 describes the proposed method. Section 5 discusses experimental results. This paper is concluded in Section 6.

\section{Preprocessing}

The preprocessing of spectra includes sky subtraction, continuum subtraction and de-noising.

Usually the spectra show a number of residual sky features in the regions of strong atmospheric emission and absorption lines. Where these are the strongest features in the spectrum, there is a danger that the correlation between the strong lines in the template and the sky residual will be greater than the correlation between the template and the much weaker spectra. We remove these sky residuals by median filter in $6 \mathrm{~nm}$ bands around $557 \mathrm{~nm}, 630 \mathrm{~nm}$ etc.

Continuum subtraction reduces the smoothly varying background to zero and essentially has the same effect as filtering out the long-period Fourier components of the spectra. Without continuum subtraction, the cross-correlation function shows a peak representing the cross-correlation of the two continua, with a small spectral cross-correlation peak superimposed. In addition, the intensities of spectral lines are not shown truly for the existence of continua. The continuum is fitted from the observed spectrum by a median filter with a filter window $60 \mathrm{~nm}$ wide. We divide the observed spectrum by the continuum, and subtract one from the spectrum.

The noise in astronomical spectra can be roughly regarded as random white noise, so soft- thresholding [5] is used for noise removal.

The middle part in fig. 2 shows the result of the preprocessing of the spectrum shown up in fig.2.

\section{$3 \quad$ Feature Extraction}

We call the spectrum after preprocessing line spectrum. Usually there are many pseudo spectral lines on line spectra because of the heavy noise contamination and the rough continuum fitting. The feature used in our method is the feature wavelengths of the emission lines. How to extract all spectral lines from line spectra is a hot potato. Spectral line extraction usually includes two steps: at first, the line spectrum is thresholded; secondly, we search for the locations of local maxima on the line spectrum, which are regarded as the feature wavelengths of spectral line candidates. Because the intensity at peak of every spectral line 
is different, it's difficult to choose an appropriate universal threshold. If the threshold is too high, it's possible that no spectral line is obtained. Conversely, if the threshold is too low, many pseudo spectral lines will be obtained. Therefore, the conjunction of local thresholding and universal thresholding by the formula (1) is adopted here.

$$
s(i)=\left\{\begin{array}{ll}
s(i), & s(i)>T(i) \& s(i)>T_{0} \\
0, & \text { else }
\end{array} \quad i=1,2, \cdots\right.
$$

Where $s(i)$ is the intensity of the $i$ th point on the spectrum, $T(i)$ is the local threshold at that point, $T_{0}$ is the universal threshold, which is the lower limit for intensities at the peak of spectral lines. We set $T_{0}=1.5 * \mathrm{rms}$, where $\mathrm{rms}=$ $\sqrt{\frac{\sum_{i=1}^{n} s(i)^{2}}{n}}$ denotes the root mean square (RMS) of the $\operatorname{array}\{s(i), i=1,2, \cdots, n\}$.

For a point on the spectrum, we take a fixed-width window centered by the wavelength at the point and compute the RMS of the intensities of all the points in the window, and choose $c * R M S$ as the local threshold for that point. By experience, the window width is $100 \mathrm{~nm}$ and $c=2.5$ in this paper. We found in experiments that the result of the feature extraction was less sensitive to the variation of the window width due to the sparse distribution of spectral lines.
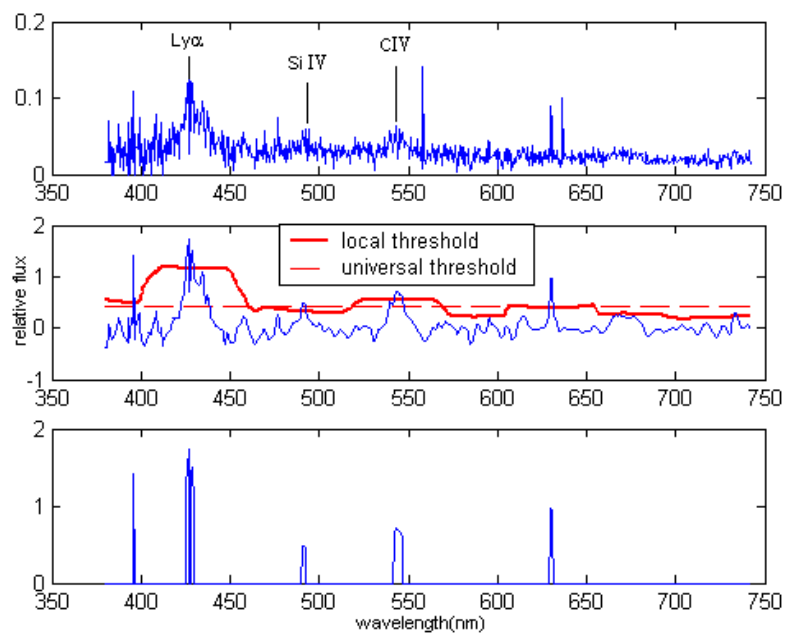

Fig. 2. Preprocessing and feature extraction (up: original spectra; middle: the spectra after preprocessing; low: spectral lines)

The thresholds are shown in the middle part of fig.2 and the emission line candidates are shown low in fig. 2 . 


\section{Redshift Determination}

\subsection{Redshift Candidate and Similarity Measure}

Let $L=\left\{\lambda_{i}, i=1,2, \ldots, N\right\}$ denote the feature wavelengths obtained from the observed spectrum. Let $L^{\prime}=\left\{\lambda_{i}^{\prime}, i=1,2, \ldots, M\right\}$ denote the feature wavelengths of all emission lines on QSO template. Set

$$
z c=\lambda / \lambda^{\prime}-1
$$

where $\lambda \in L, \quad \lambda^{\prime} \in L^{\prime}$.

Definition 1: If $z c \geq 0$, we define $z c$ as a redshift candidate of the observed spectrum.

Let $d$-dimensional vector $X$ and $Y$ denote spectra $\mathrm{A}$ and $\mathrm{B}$ respectively. We evenly divided the two spectra into $K$ parts. Let $X=\left[X_{1}, \cdots, X_{K}\right]$ and $Y=\left[Y_{1}, \cdots, Y_{K}\right]$. Set

$$
r_{A B}=\sum_{i=1}^{K} w_{i}\left(X_{i} Y_{i}^{T}\right) /\left(\left\|X_{i}\right\|\left\|Y_{i}\right\|\right)
$$

Where $\sum_{i=1}^{K} w_{i}=1, w_{i}$ denotes the weight.

Definition 2: Define $r_{A B}$ as the similarity measure between spectra $A$ and $B$.

\subsection{Redshift Determination}

Redshift determination based on similarity measure is composed of the following four steps:

Step1: Perform the continuum subtraction for the QSO template.

Step2: Determine the redshift candidates of the observed spectrum by formula (2).

Step3: Shift the QSO template after continuum subtraction according to every redshift candidate and compute the similarity between the shifted template and the observed spectrum of the sky and continuum subtraction.

Step4: Choose the redshift candidate corresponding to the highest similarity as the redshift of the observed spectrum.

Remark1: The proposed similarity measure becomes the traditional one with $K=1$. Because the observed spectra always vary drastically from the rest frame, if the similarity is measured traditionally, it's possible that the similarity corresponding to the redshift is lower than others due to the high local correlation caused by noise disturbance or by the two strong lines corresponding to some redshift candidates. Similar to the evidence theory [6], the similarity measure proposed is the weighted sum of several similarity evidences. The principle of setting the weights is that the smaller the angle between $X_{i}$ and $Y_{i}$, the higher the weight $w_{i}$. 


\subsection{Comparison with the Traditional Cross-Correlation Based Methods}

This method is different from the traditional cross-correlation based methods (here called CCM) in the following aspects. Firstly, CCM is based on the least squared criterion, so it requires the wavelength range of the templates and the observed spectrum must be identical, while our method has not the limitation because it only needs to shift the template according to the redshift candidates. Due to the limitation, CCM only can be used for low redshift spectra (generally with redshifts lower than 0.3 ), while our method also can be used for high redshift spectra. Secondly, in fact, the cross-correlation in CCM is measured for the samples produced by uniform sampling in a redshift interval, which can be regarded as the redshift candidates in our method. It's evident that the number of the redshift candidates is smaller in our method than in CCM. Because the value corresponding to the redshift is not necessarily the highest peak of the cross-correlation function for those spectra with high noise contamination, the error risk is lower in our method than in CCM.

\section{$5 \quad$ Experiments}

In this section, both simulated spectra and observed spectra are used to verify the effectiveness of the proposed method. The QSO template, as shown in fig.3, is from Vanden Berk et al [7]. Because we can't get the templates used in $\mathrm{CCM}$, in all the subsequent experiments, we don't report the comparison with it. The density estimation method (called DEM) is a typical method based on spectral line matching. It includes the following steps: firstly, the spectral lines are extracted and used to determine the redshift candidates; secondly, the densities of the candidates are estimated; finally, the average of the candidates in a neighborhood of the candidate with maximal density is regarded as the redshift. The comparisons with DEM are reported. In the experiments, the spectra are divided into four segments for the similarity measure, and the weight is set to be $0.1: 0.2: 0.3: 0.4$ under the principle in the remark 1 .

\subsection{Experiment with Simulated Spectra}

In this experiment, the simulated spectra are generated by shifting the QSO template with redshift values ranging from $0-2$ with a step of 0.01 . All simulated spectra are digitized and linearly interpolated to the wavelength range of $380-$ $742 \mathrm{~nm}$ with a step of $0.5 \mathrm{~nm}$.

A Guassian noise with 0 mean and $\sigma$ standard deviation (noise level) is added to the spectra. The correct rate vs. $\mathrm{SNR}(\mathrm{SNR}=1 / \sigma)$ is plotted in fig.4. All the results in fig. 4 are the average value of 100 independent trials. It can be seen from the figure that the correct rate is increased with the raising of SNR and the correct rate reaches $97 \%$ above at the SNR of 6 . This indicates that the proposed technique is robust and effective. 
For a comparison with DEM, the corresponding result from DEM is also plotted in fig.4. We clearly see that the two methods are incomparable at every noise level. This is mainly because there are few spectral lines on the QSO spectra when redshifts are larger than 0.5, and it is difficult for a method based on spectral line matching to determine the redshift correctly under this condition.

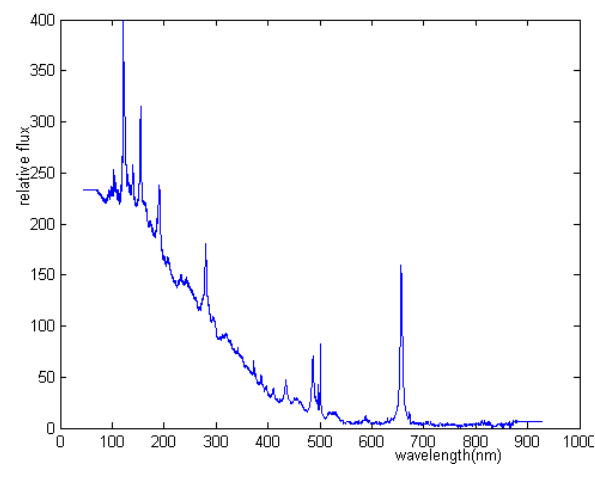

Fig. 3. The quasar template

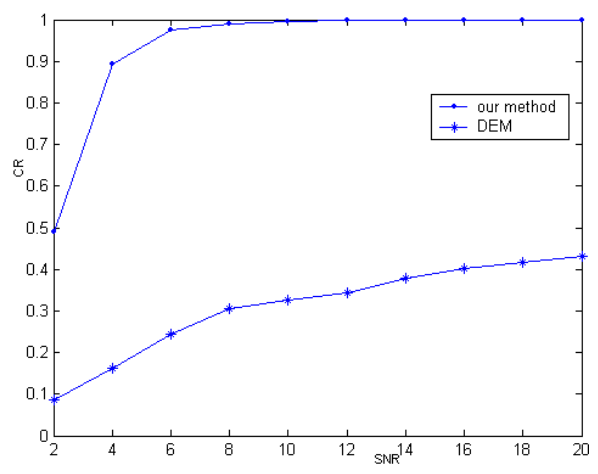

Fig. 4. Correct rate vs. SNR

\subsection{Experiments with Observed Spectra}

In the following experiments, the test data includes 3056 observed QSO spectra from SDSS data release2. The redshifts of these test spectra vary from 0 to 5 .

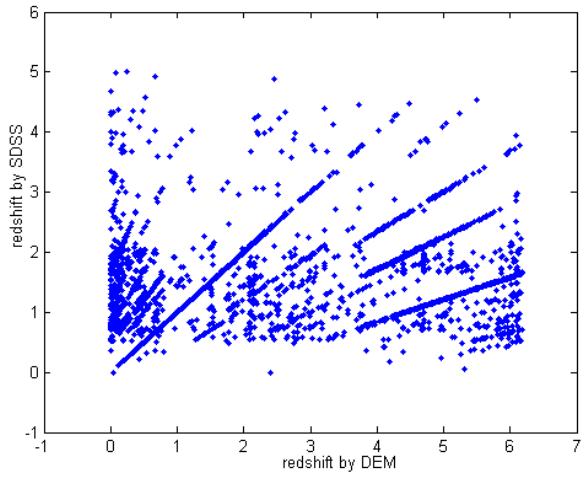

Fig. 5. DEM

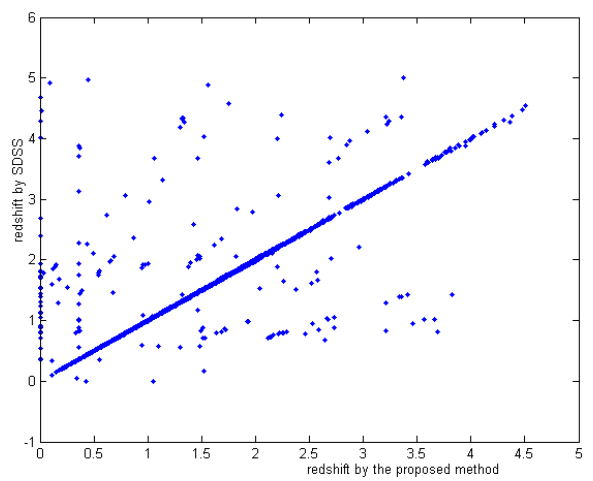

Fig. 6. The proposed method

Comparisons with DEM: Fig.5 shows the results from DEM. Fig.6 is from the proposed method. The horizontal axes denote the results obtained by the two methods, and the vertical axes denote the redshifts from SDSS. The points on 
the line $(y=x)$ denote the obtained redshifts are coincident with the redshifts from SDSS. The correct rates in Figs. 5, 6 are $24.8 \%$ and $94.2 \%$ respectively. The results show that the proposed method is superior to DEM. From the fig.5, we can see that the error is mainly for those spectra with redshifts larger than 0.5. This indicates DEM is also not fit for high redshift spectra.

All methods based on spectral line matching have a high requirement for the quality of the spectral line extraction. When there are more pseudo spectral lines and fewer spectral lines in the result of spectral line extraction, it's difficult to get the right results for redshift determination. Comparatively, the quality of the spectral line extraction has a lower effect on our method. In principle, even if there is only one spectral line in the result of spectral line extraction, the redshift can be determined correctly. However, it's unlikely that every observed spectrum agree with the template. As a whole, the proposed method overcomes the defect of the methods based on spectral line matching.

Table 1. The correct rates under different tolerated error by different strategies

\begin{tabular}{|l|l|l|l|l|l|l|l|}
\hline & $K=1$ & $K=2$ & $K=3$ & $K=4$ & $K=5$ & $K=6$ & $K=7$ \\
\hline$\varepsilon=0.01$ & $83.5 \%$ & $86.1 \%$ & $87.4 \%$ & $87.1 \%$ & $86.7 \%$ & $85.9 \%$ & $85.1 \%$ \\
\hline$\varepsilon=0.02$ & $89.5 \%$ & $91.8 \%$ & $93.1 \%$ & $93.2 \%$ & $92.5 \%$ & $92.1 \%$ & $91.7 \%$ \\
\hline$\varepsilon=0.03$ & $90 \%$ & $92.4 \%$ & $94 \%$ & $94 \%$ & $93.5 \%$ & $93.1 \%$ & $92.5 \%$ \\
\hline
\end{tabular}

Comparisons Among Different Similarity Measure Strategies: In this part, different similarity measure strategies are compared. We evenly divide the spectra into $K=1 \sim 7$ parts respectively. The weights are chosen as $1 / K$ in every similarity measure. Tab. 1 shows the correct rates under different tolerated error $\varepsilon$. From the Tab.1, we can see that under every tolerated error, the correct rate is increased with the $K$ varying from 1 to 3 , while it is decreased with the $\mathrm{K}$ varying from 4 to 7 . The result by the traditional similarity measure $(K=1)$ is the weakest. This proves the proposed similarity measure is more effective and reasonable than the traditional one.

\section{Conclusion}

Up to now, nearly all the existing methods of redshift determination are for galaxy spectra with small redshifts, there hasn't been a successful technique for QSO spectra published in the literature. In this paper, we presented a new cross-correlation method for redshift determination of QSO spectra based on similarity measure. The similarity measure proposed, which is similar to the evidence theory, is more reasonable than the traditional ones. The proposed method overcomes the defect that high redshift spectra can't be processed by the traditional cross-correlation based methods. Compared with the methods based on spectral line matching, our method is less sensitive to the quality of spectral line extraction. Experiment results have demonstrated the proposed 
method is robust and effective. We think that the idea of the similarity measure proposed can also be used in other areas that need similarity measure. Further works will be dedicated to confidence analysis and error estimation.

\section{Acknowledgement}

This work is supported by the National 863 High Technology R\&D Program under grant No.2003AA133060.

\section{References}

1. John.Tonry, Marc.Davis : A Survey of Galaxy Redshifts. I. Data Reduction Techniques. The Astronomical Journal. 58(10) (1979) 1511-1525

2. Karl.Glazebrook, Alison.R.Offer, Kathryn.DeeleyJ : Determination By Use of Principal Component Analysis. I. Fundamentals. The Astronomical Journal. 492(1) (1998) 98-109

3. L Y. Huang, F. M. Sun, Z Y. Hu : A new automatic quasar recognition technique based on PCA and the Hough Transform. ICPR2000. 2 (2000) 499-502

4. DUAN Fuqing, WU Fuchao, LUO Ali et al: Density estimation based model matching method for redshift determination Spectroscopy and Spectral Analysis (in Chinese)(to appear)

5. D.L Donoho : De-noising by soft-thresholding. IEEE Trans.on IT. 41(3) (1995) 613-627

6. J. W. Guan, D. A. Bell. Evidence theory and its applications. Elsevier Science Ltd, New York, USA. (1991)

7. DE Vanden Berk, GT Richards, et al : Composite quasar spectra from the Sloan Digital Sky Survey. The Astronomical Journal. 122(8) (2001) 549-564 